\title{
On the Number of Ordinary Circles Determined by $n$ Points
}

\author{
Ruixiang Zhang
}

Received: 17 February 2010 / Revised: 29 May 2010 / Accepted: 14 July 2010 /

Published online: 11 September 2010

(C) Springer Science+Business Media, LLC 2010

\begin{abstract}
In this paper we deal with the following problem: Given a set $B$ consisting of $n$ points, not all on a line or a circle. A circle passes through exactly three points of $B$ is called an ordinary circle. What is the minimal possible number of ordinary circles determined by $B$ ? In this paper we improve the best-known lower bound $\frac{22}{247}\left(\begin{array}{l}n \\ 2\end{array}\right)$ to $\frac{1}{9}\left(\begin{array}{l}n \\ 2\end{array}\right)$.
\end{abstract}

Keywords Ordinary line · Ordinary circle

\section{Introduction}

More than a hundred years ago, Sylvester [7] posed the following problem: Is it true that any finite set of points in the Euclidean plane, not all on a line, has two elements whose connecting line does not pass through a third? In the 1930s, the question was rediscovered by Erdős and shortly afterwards, an affirmative answer was given by Gallai. Besides, many alternative proofs and generalizations were given. For more detailed information about this topic, see [2].

Naturally, the next task was to find the minimal number ol $(n)$ of ordinary lines (lines that pass through precisely two points) determined by $n$ noncollinear points in the plane. Kelly and Moser [5] showed that $o l(n) \geq \frac{3}{7} n$. The best lower bound up to now was given by Csima and Sawyer [3], who showed that $o l(n) \geq \frac{6}{13} n$ except for the Kelly-Moser configuration.

Still, there are many interesting problems of this type for curves other than straight lines. In this paper, we deal with the following problem of Sylvester-Gallai-type: A circle is called ordinary if it passes through precisely 3 elements of the underlying set. Given a set $B$ of $n$ noncollinear points in the plane, not all on a circle

R. Zhang $(\varangle)$

School of Mathematical Sciences, Peking University, Beijing 100871, P.R. China

e-mail: zrx_gpx@126.com 
either. What is the minimal number of ordinary circles determined by them? The earliest researcher who concerned about the analogue for ordinary circles I know was Motzkin [6]. And Elliott [4] was the earliest one I know to ask the question of the minimal number of ordinary circles. The lower bound he gave was $\frac{4}{63}\left(\begin{array}{c}n \\ 2\end{array}\right)$. According to Brass et al. [2], $\frac{22}{247}\left(\begin{array}{l}n \\ 2\end{array}\right)$, the best lower bound known so far, was given by Bálintová and Bálint [1]. In this paper, by considering the problem in the real projective plane, adopting some ideas used by Kelly and Moser [5] and showing a key theoremTheorem 4.1 in this paper (and pointing out that in a certain sense its result is the best), we improve the bound to $\frac{1}{9}\left(\begin{array}{l}n \\ 2\end{array}\right)$.

\section{Definitions and Preliminary Results}

Here we make a short review of some definitions and theorems given by Kelly and Moser [5] which will be used in the following discussion. Let $P$ be a set of $m$ noncollinear points in the real projective plane and $S$ the set of connecting lines (a line is called connecting if it passes through at least two points of $P$ ). A generic point of $P$ is denoted by $p$ and a generic line of $S$ by $s$. Subscripts distinguish particular points and lines.

For the precise definitions of residence (one of the regions dissected by the lines that do not pass through $p$, in which $p$ lies), neighbor (the lines containing the edges of the residence) or near-pencil (a set of lines in the plane such that all are concurrent except one line), which are used in this paper, see [5].

Next we list some preliminary theorems, among which Theorems 2.1, 2.2 and 2.3 have been proven by Kelly and Moser [5] as Theorem 2.3, Corollary 3.4 and Theorem 3.1, respectively in their paper. As for the proof of our Theorem 2.4, see the proof of Theorem 3.1 in Kelly and Moser's paper [5]. Our theorem is a direct corollary of it.

Theorem 2.1 If $S$ is not a near-pencil, each point of $P$ has at least three neighbors.

Theorem 2.2 A line of $S$ is a neighbor of at most four points.

Let $q$ be an arbitrary point in the plane and suppose $q \notin P$. We call the number of ordinary lines passing through $p$ the order of $p$, the number of ordinary lines passing through $p$ but not through $q$ the non- $q$ order of $p$. The number of neighbors of $p$ which are ordinary lines is the rank of $p$ and the number of neighbors of $p$ which are ordinary lines that do not pass through $q$ the non- $q$ rank of $p$. The order plus the rank is the index and similarly we have the definition of non- $q$ index.

Theorem 2.3 If the order of $p$ is 0 then every neighbor of $p$ is an ordinary line.

Theorem 2.4 If a neighbor $s$ of $p$ is one that passes through three points of $P$, say $p_{1}, p_{2}$ and $p_{3}$, and there is a point $x \in s$ on the boundary of the residence of $p$ such that $p_{1} x / / p_{2} p_{3}$ (that is, $p_{1}$ and $x$ separate $p_{2}$ and $p_{3}$ ) then line $p p_{1}$ is ordinary. 


\section{On the Non- $q$ Index}

The following is our key lemma, which shows that in most cases the non- $q$ index of $p$ is at least 1 .

Theorem 3.1 As long as $S$ is not a near-pencil, the non-q index of $p$ is at least 1.

Proof We have 3 different cases to examine. For different possible order of $p$, we will use different techniques accordingly.

Case 1. The order of $p$ is 0 . By Theorem 2.3, all the neighbors of $p$ are ordinary. By Theorem 2.1, $p$ has at least three neighbors which obviously can not be concurrent (otherwise they would not all be the neighbors of $p$ ) and so can not all pass through $q$. Therefore the non- $q$ rank of $p$ is at least 1 and so is the non- $q$ index.

Case 2. The order of $p$ is at least 2. Note that two different ordinary lines that both pass through $p$ cannot both pass through $q$, we deduce the non- $q$ order of $p$ is at least 1 and so is the non- $q$ index.

Case 3. The order of $p$ is precisely 1 . We assume $s$ is the only ordinary line that passes through $p$ and it also passes through another point $p_{0} \in P$. If the non- $q$ order or the non- $q$ rank of $p$ is positive, then the non- $q$ index of $p$ is also positive and Theorem 3.1 holds. Now we assume that both the non- $q$ order and the non- $q$ rank of $p$ are 0 .

If a neighbor of $p$ is ordinary, we call it an ordinary neighbor (of $p$ ). Otherwise we call it a special neighbor. Note that we have shown that any three neighbors of $p$ cannot be concurrent in the discussion of Case 1, we deduce that no more than 2 neighbors of $p$ can pass through $q$. Since the non- $q$ rank of $p$ is 0 , there can be no more than 2 ordinary neighbors of $p$. By Theorem 2.1, $p$ has at least 1 special neighbor.

For an arbitrary special neighbor $\bar{s}$ of $p$, suppose it passes through three points of $P$. Denote the three points by $p_{1}, p_{2}$ and $p_{3}$, respectively.

If, among the three points, there is a point, say, $p_{2}$ is on the boundary (excluding vertices) of the residence of $p$, then we can choose two points $x_{1} \in \bar{s}$ and $x_{3} \in \bar{s}$ which are both on the boundary of the residence such that $x_{1} p_{1} / / p_{2} p_{3}$ and $x_{3} p_{3} / / p_{1} p_{2}$, as is shown in Fig. 1(a). By Theorem 2.4 the lines $p p_{1}$ and $p p_{3}$ are both ordinary. However we have assumed that the order of $p$ is 1 . A contradiction.

Therefore neither of $p_{1}, p_{2}$ and $p_{3}$ is on the boundary (excluding vertices) of the residence of $p$ (but some of them might be vertices of the residence). Without loss of generality, we suppose that, as is shown in Fig. 1(b), for an arbitrary point $x \in \bar{s}$ on the boundary of the residence of $p$,

$$
x p_{1} / / p_{2} p_{3} .
$$

By Theorem 2.4 we deduce that line $p p_{1}$ is the unique ordinary line passing through $p$ (note that the order of $p$ is 1 ) and $p_{1}=p_{0}$. By (1) we learn that $p_{0}$ does not lie on the boundary (including vertices) of the residence of $p$.

Because in the above discussion the special neighbor $\bar{s}$ is arbitrarily chosen, one can easily see that each of the special neighbors passes through $p_{0}$. Again since any 


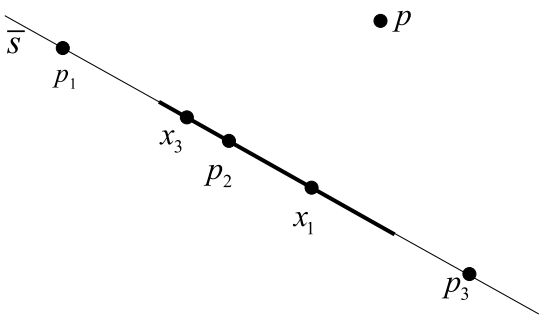

(a)

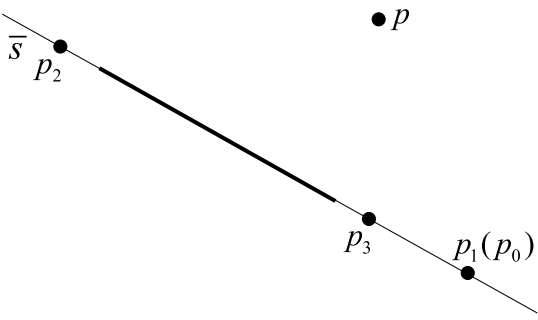

(b)

Fig. 1 The bold line is the common part of line $\bar{s}$ and the boundary of the residence of $p$

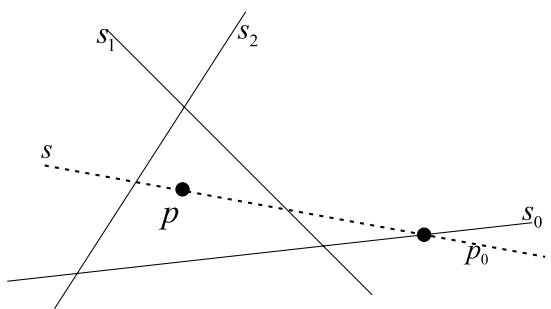

(a)

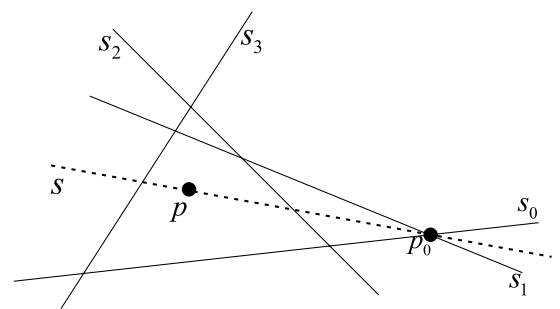

(b)

Fig. 2 The three lines $s_{1}, s_{2}$ and $p p_{0}$ cannot be concurrent in part (a) and the three lines $s_{2}, s_{3}$ and $p p_{0}$ cannot be concurrent in part (b)

three neighbors of $p$ cannot be concurrent, we know that there can be at most two special neighbors of $p$. We have the following two subcases.

Subcase 1. There is precisely 1 neighbor of $p$ which is special. Since we have shown that, under our assumption that the non- $q$ rank of $p$ is 0 , the point $p$ can have at most two ordinary neighbors, by Theorem 2.1 we deduce that $p$ has two ordinary neighbors and one special neighbor and we have a triangular residence. Suppose the two ordinary neighbors are $s_{1}$ and $s_{2}$. Because we have supposed the non- $q$ rank of $p$ is 0 , three lines $s_{1}, s_{2}$ and $p p_{0}$ must all pass through $q$ and therefore be concurrent. However since $p_{0}$ does not lie on the boundary (including vertices) of the residence of $p$, the above three lines cannot be concurrent (see Fig. 2(a)). A contradiction.

Subcase 2. There are precisely two neighbors of $p$ which are special. Denote them by $s_{0}$ and $s_{1}$. We have shown that both $s_{0}$ and $s_{1}$ pass through $p_{0}$. However we also have proven that $p_{0}$ does not lie on the boundary (including vertices) of the residence of $p$. With this in mind and note that $p$ have no more than two ordinary neighbors, we deduce that $p$ must have four neighbors, among which two are ordinary (denoted by $s_{2}$ and $s_{3}$ ) while the other two special, and the residence of $p$ is quadrilateral. Also, $s_{0}$ and $s_{1}$ must be opposite sides of the residence (otherwise we still have $p_{0}$ on the boundary, including vertices, of the residence if they are adjacent), as is depicted in Fig. 2(b). However, in this case three lines $p p_{0}, s_{2}$ and $s_{3}$ cannot be concurrent. A contradiction. 
Fig. 3 In this case,

$P=\left\{p_{i} \mid 1 \leq i \leq 6\right\}$. The dotted lines are the only three ordinary lines. Clearly, there is only one ordinary line that does not pass through $q$. That is to say, in this figure there is precisely $\frac{1}{6} m$ non- $q$ ordinary line

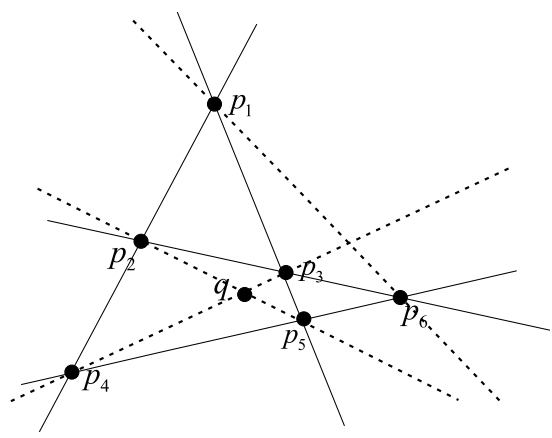

Therefore our assumption at the beginning of the discussion of this case that both the non- $q$ order and the non- $q$ rank of $p$ are 0 cannot be true, which proves the theorem in this case.

\section{Non- $q$ Ordinary Lines and Ordinary Circles}

If an ordinary line of $S$ does not pass through $q$, we call it a non- $q$ ordinary line. The following theorem gives a lower bound of the total number of non- $q$ ordinary lines.

Theorem 4.1 There exist at least $\frac{1}{6} m$ non-q ordinary lines.

Proof Since the points of $P$ are not collinear, we have $m \geq 3$.

If $S$ is a near-pencil, it can be easily seen that there are $(m-1)$ ordinary lines in $S$ which are concurrent at a point $p \in P$. Since $q \notin P$, among the $(m-1)$ lines there are at least $(m-2)$ lines that do not pass through $q$. Because $m \geq 3$, we have $m-2 \geq \frac{1}{6} m$ and Theorem 4.1 holds.

Now we assume that $S$ is not a near-pencil. Consider the problem in real projective plane. By Theorem 3.1, the non- $q$ index of any point $p$ in $P$ is not less than 1 . So the sum of all those non- $q$ index is at least $m$. On the other hand, any ordinary line $s$ that do not pass through $q$ can make contribution to this sum no more than six times (note that it only contributes to the non- $q$ order of two points of $P$ on $s$, and can contribute to the non- $q$ rank of at most four points of $P$ by Theorem 2.2). Therefore it is clear that there exist at least $\frac{1}{6} m$ non- $q$ ordinary lines and Theorem 4.1 holds.

In Fig. 3, we can see that in a certain sense Theorem 4.1 gives the best possible bound.

The following is our main theorem and finally we can show it.

Theorem 4.2 Given a set $B$ of $n$ noncollinear points in the plane. Suppose the points of $B$ are not all on a circle either. A circle is called ordinary if it passes through precisely three points of $B$. Then the number of ordinary circles is not less than $\frac{1}{9}\left(\begin{array}{l}n \\ 2\end{array}\right)$.

Proof First we show that for an arbitrary point $b \in B$, there are at least $\frac{1}{6}(n-1)$ ordinary circles that pass through $b$. In fact this can be easily obtained by inversion. 
Let $\Omega$ be a circle of inversion which center $b$ and $\overline{B \backslash\{b\}}$ be the inverse of $B \backslash\{b\}$. Because the points of $P$ are not all on a line or on a circle, the points of $\overline{B \backslash\{b\}}$ are noncollinear. By Theorem 4.1, there exist at least $\frac{1}{6}(n-1)$ connecting lines of $\overline{B \backslash\{b\}}$ that are ordinary and do not pass through $b$. All of them are transformed by inversion with respect to $\Omega$ into ordinary circles passing through $b$.

Note that each of the ordinary circles passes through precisely three points in $B$, by the method of double-counting we can easily deduce that there exist at least $\frac{1}{9}\left(\begin{array}{l}n \\ 2\end{array}\right)$ ordinary circles.

\section{An Upper Bound and Conclusion}

Up to now, we have got a lower bound of the least possible number of ordinary circles. However, it is still far from optimal. In fact, as for the upper bound, the best result I can come up with is the following theorem.

Theorem 5.1 Let $B$ be a set of $n(>4)$ points in the plane that are not all on a line or a circle. Let oc $(n)$ be the least possible number of ordinary circles of B. Then oc $(n)$ satisfies the following inequality:

$$
o c(n) \leq \begin{cases}\frac{(n-1)(n-3)}{2}, & 2 \nmid n, \\ \frac{n(n-2)}{4}, & 2 \mid n \text { but } 4 \nmid n, \\ \frac{n(n-4)}{4}, & 4 \mid n .\end{cases}
$$

Proof It is sufficient to give the examples to finish the proof. Let $B$ be on the complex plane. If $n$ is odd, let

$$
B=\{0\} \cup\left\{e^{\frac{2 \pi i}{n-1}} \mid 0 \leq i<n-1\right\} .
$$

If $n$ is even, let

$$
B=\left\{e^{\frac{4 \pi i}{n}} \mid 0 \leq i<\frac{n}{2}\right\} \cup\left\{2 e^{\frac{4 \pi i}{n}} \mid 0 \leq i<\frac{n}{2}\right\} .
$$

Note that $n>4$, one can easily show that the points of $B$ are noncollinear and not on a circle. Moreover, it is not hard to prove the number of ordinary circles of $B$, denoted by $o c(B)$, satisfies

$$
o c(B)= \begin{cases}\frac{(n-1)(n-3)}{2}, & 2 \nmid n, \\ \frac{n(n-2)}{4}, & 2 \mid n \text { but } 4 \nmid n, \\ \frac{n(n-4)}{4}, & 4 \mid n .\end{cases}
$$

And the theorem has been proved.

Theorem 5.1 tells us some information about the upper bound of $o c(n)$. However, as it can best be approximately $\frac{n^{2}}{4}$ and at other time it is about $\frac{n^{2}}{2}$, the lower bound 
given by our Theorem 4.2 (which is approximately $\frac{n^{2}}{18}$ ) is not likely to be the best. Elliott [4] conjectured that it should be about $\frac{n^{2}}{6}$. But he did not provide much further clues about how he conjectured it in his paper. So deciding the approximate value of $o c(n)$ still remains to be an open problem.

In my proof, some techniques in Kelly-Moser paper were adopted. In 1993, Csima and Sawyer [3] improved the Kelly-Moser result in condition of $n>7$. Unfortunately, my technique of the proof in this paper seems not be able to explore this fact.

Acknowledgements I would like to thank Professor János Pach and the referees for many helpful advices on this paper.

\section{References}

1. Bálintová, A., Bálint, V.: On the number of circles determined by $n$ points in the Euclidean plane. Acta Math. Hung. 63(3), 283-289 (1994)

2. Brass, P., Moser, W., Pach, J.: Research Problems in Discrete Geometry, pp. 302-310. Springer, Berlin (2005)

3. Csima, J., Sawyer, E.T.: There exist 6n/13 ordinary points. Discrete Comput. Geom. 9(2), 187-202 (1993)

4. Elliott, P.D.T.A.: On the number of circles determined by $n$ points. Acta Math. Acad. Sci. Hung. 18, 181-188 (1967)

5. Kelly, L.M., Moser, W.O.J.: On the number of ordinary lines determined by $n$ points. Can. J. Math. 10, 210-219 (1958)

6. Motzkin, T.S.: The lines and planes connecting the points of a finite set. Trans. Am. Math. Soc. 70, 451-464 (1951)

7. Sylvester, J.J.: Mathematical question 11851. Educ. Times. 46, 156 (1893) 